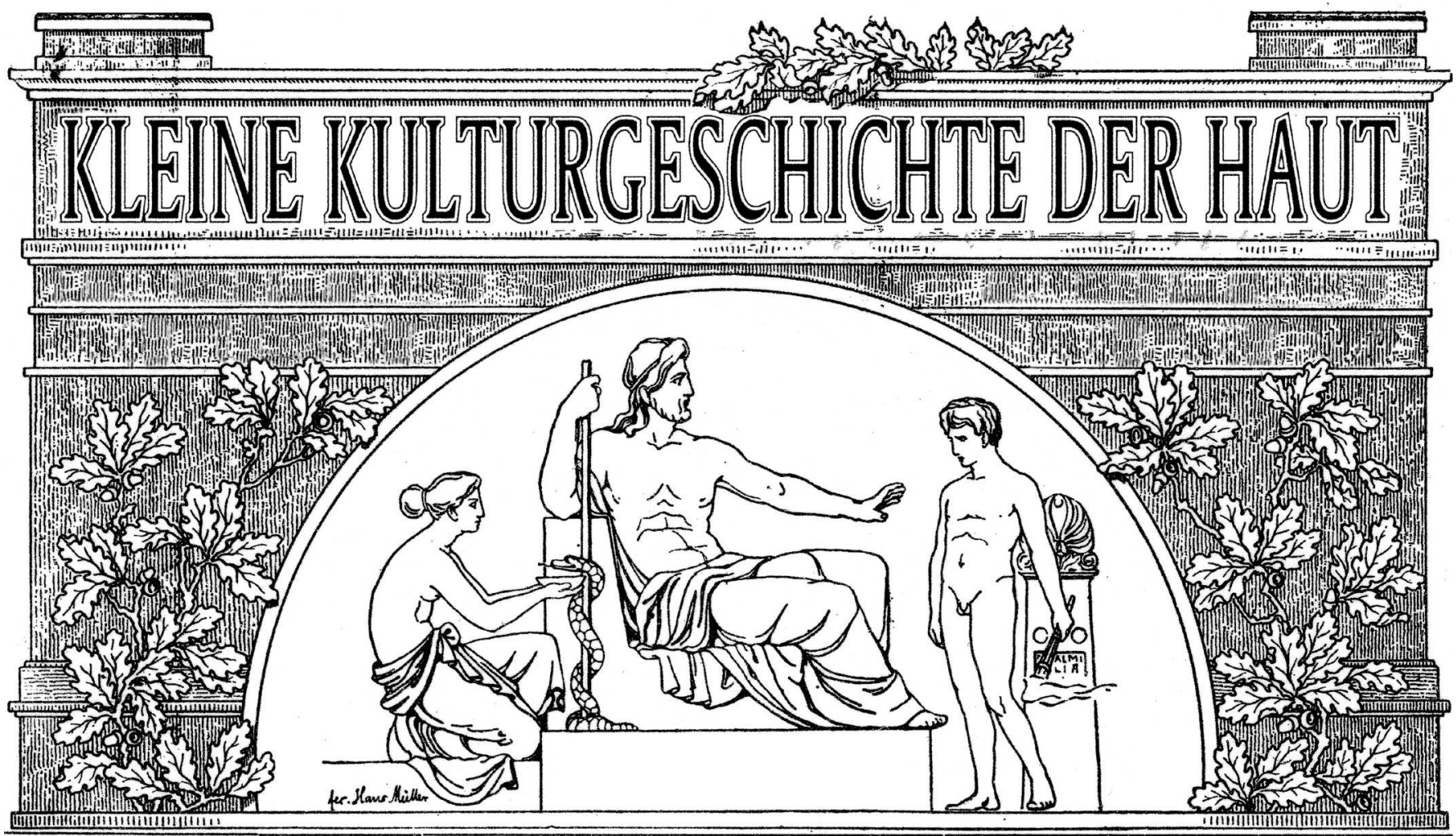

Die Zeitschrift „Aktuelle Dermatologie“ hat mit Beginn des Jahres 2004 diese Rubrik neu geöffnet, um in loser Folge kurze, prägnante und informative Artikel zu publizieren zu vielen Aspekten der Kulturgeschichte in Beziehung zur Haut. Dies erstreckt sich auf alle Kulturen und Zeiten, auf die Künste, auf Mythen und Psychologie und natürlich auch auf medizinische Aspekte.
Unsere Hoffnung, dem geneigten Leser Interessantes zu bieten, den Blick zu weiten und Freude zu bereiten, hat sich auf das Trefflichste bewährt. Reichlich Zusprüche, Kommentare, Vorschläge und aktive Teilnahme haben uns erreicht und erfreut. Dies möge weiter so gehen. Und so werden auch wir fleißig bemüht bleiben, Sie zu erbauen mit unserer kleinen Kulturgeschichte der Haut

\title{
Sonne und Sonnenkult
}

\section{E. G. Jung}

Die Sonne spendet Licht und Wärme und sie ist die wesentliche Energiequelle für die Biosphäre unserer Erde. Dies wurde in allen Kulturen und antiken Religionen erfasst. Ihr kommt auch ein gewichtiger Symbolwert zu. Neben dem Symbol des Lebens an sich, ist sie auch ein ganz frühes Symbol für Richtung, für die Ausrichtung in der geordneten Welt, denn sie geht im Osten auf und im Westen unter. „Wegweiser“ wurde sie genannt. Die Sonne ist aber auch ein Symbol des Rhythmus, da sie den Takt von Tag und Nacht angibt als „Teiler der Zeit“.

Die Sonne ist ein männliches Symbol und wird verkörpert, als ein Held, der unermüdlich und immer wieder gegen die Finsternis ankämpft. In den frühen polytheistischen Religionen mit Naturgöttern nimmt sie als Obergottheit eine besondere Stellung ein und stellt ein Leitsymbol patriarchalischer Religionsphasen dar. Diese entsprachen den Sozialstrukturen der sesshaften Siedler, die als
Pflanzer, Sammler und Jäger zwar ausziehen, immer aber wieder an ihren Stammplatz zurückkehren [1].

Die Sonnenreligionen haben die noch älteren Religionen matriarchalischer Ausrichtung mit der Muttergöttin Erde oder der ebenfalls weiblichen Mondgöttin abgelöst. Und sie sind wiederum abgelöst worden von Religionen mit einer Vielzahl von Göttern mit teils menschlichen, teils idealisierten Eigenschaften, wie die olympische Götterwelt Griechenlands oder diejenige im alten Rom. So ist Helios als Sohn des Titanen Hyperion nicht von Zeus' Geschlecht, welches dasjenige der Titanen beherrschte und ablöste.

Die Sonne ist ein Symbol des aktiven, gegen die Finsternis kämpfenden Menschen, der im Gegensatz zu den Nomaden, an seinen Sitz zurückkehrt. Sie verkörpert das Besondere der Wiederkehr, welche die Sesshaftigkeit der Völker und ihrer Götter ausmacht. Am Morgen geht die Sonne im Osten auf, eine Geburt symbolisierend, und sie wandert ständig kämpfend über das Firmament, wird von Tieren auf einem Wagen gezogen, um am Abend im Westen unterzugehen. Damit wird Vergänglichkeit und Tod symboli- 
siert. In vielen Religionen wird die Sonne am Abend im Westen von einem großen Tier gefressen. Sie durchwandert während der Nacht die Unterwelt, das Jenseits oder ein Schattenreich, um am Morgen im Osten angelangt wieder aufzugehen. Dies bedeute Wiedergeburt, Neugeburt oder „Auferstehung“. Der rhythmische Sonnenlauf über den Tag und die Nacht wird in allen Religionen ähnlich geschildert und erscheint auch im Alten Testament der Bibel, wenn David im Psalm 19 singt: „Dort hat er der Sonne ein Zelt gesetzt, und sie, wie ein Bräutigam geht sie hervor aus ihrer Kammer, läuft freudig wie ein Held die Bahn. Sie geht auf an einem Ende des Himmels und geht um bis wieder an das Ende, nichts bleibt vor ihrer Glut verschont.“ [2]

Der Sonnenlauf hat aber noch andere Besonderheiten von großer Symbolkraft und Bedeutung: die Finsternisse. Die Sonnenreligionen haben in diesen die Begegnung von Sonne und Mond, also das Aufeinanderprallen des patriarchalischen mit dem matriarchalischen Prinzip gesehen. Dies kann einerseits eine fruchtbare Begegnung sein und die Befruchtung oder die Begattung symbolisieren, andererseits aber auch die Dominanz der Sonnenreligionen darstellen. Es kommt zur Verschmelzung oder Beherrschung der früheren matriarchalischen Religionen, was für Letztere das Beherrschtwerden, das Aufgefressenwerden und somit eine Katastrophe bedeutet.

Nur einmal ist es im alten Ägypten von 1364-1348 v. Chr. zur kurzen Phase einer monotheistischen Sonnenreligion gekommen. Der frühere Sonnengott Rè, einer von vielen Göttern, ist wie alle anderen abgelöst worden durch den alleinigen und einzigen Sonnengott Aton (Lichtberg), der von seinem Priesterkönig Echnaton (Sohn des Aton) verkündet und verehrt wurde (Abb.1). Ruinen, Symbole und Darstellungen sind in der Amarna-Kultur überliefert.

In den monotheistischen Erlöserreligionen, im Judentum und besonders im Christentum, ist die Sonne als Besonderheit nicht verloren gegangen. Sie stellt keinen eigenständigen Gott mehr dar. Vielmehr wird sie von dem unpersönlichen, nicht sichtbaren und zeitlosen Gott abgelöst und sie ist, wie fast alles, was das Christentum von früheren Religionen übernommen hat, in seinen Dienst genommen und zum Lobe Gottes eingesetzt worden. Im Jahre 311 n. Chr. wurde das Christentum von Kaiser Konstantin I d. Gr. (280-337 n. Chr.), der wohl unter dem Einfluss des väterlichen Sonnenkultes im Jahre 310 eine „Sol-Apollo-Vision“ hatte, toleriert und am Konzil von Nizäa 325 als Staatsreligion im römischen Reich eingesetzt. Die „allein selig machende“ christliche Kirche etabliert sich mit dem Papst in der Nachfolge Petri in Rom und strebt, getragen vom Missionsauftrag, nach globaler Dominanz. Damit wird die Erde zum „auserwählten“ Planeten, zum Mittelpunkt der damaligen Welt, um welchen sich die Gestirne und auch die Sonne (geozentrisches System), zu drehen haben.

Die Sonne wird denn auch im Mittelalter wiederholt in den Dienst der Lobpreisung Gottes gestellt. Franziskus von Assisi, der Gründer des Franziskaner-Ordens, besingt 1224 in seinem Sonnengesang (Cantioco di Frate Sole) den Bruder Sonne und die Schwester Mond und beschwört beide, ihn in der Lobpreisung Gottes zu unterstützen. Ähnliches hat der Dominikaner-Mönch Tommaso Campanella 1602 ausgedrückt, als er im Gefängnis seinen utopischen Roman „Città del Sole“ schrieb und einen idealisierten Sonnenstaat beschrieb.

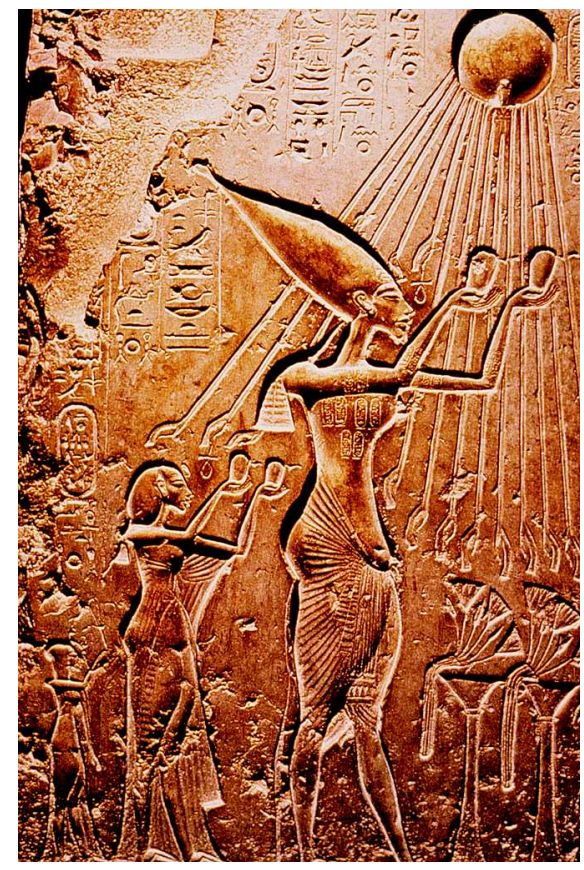

Abb. 1 AmarnaKultur in Ägypten (1364-1348 v. Chr.). Menschen und Pflanzen empfangen die Leben spendenden Sonnenstrahlen und der Priester-König Echnaton huldigt dem allein herrschenden Sonnengott Aton.

Im seinem Todesjahr veröffentlicht Nikolaus Kopernikus (1473-1543) auf Grund von Beobachtungen und Messungen ein „heliozentrisches“ Weltsystem, welches das geozentrische ptolemäische System ablöste (Kopernikanische Wende). Allerdings wurde die Arbeit zunächst als Denkmodell abgetan, bis 1632 Galileo Galilei (1564-1642) in seiner berühmten Schrift „Dialog über die beiden Weltsysteme, das ptolemäische und das kopernikanische“ der heliozentrischen Ansicht zum Durchbruch verhalf. Ursprünglich genehmigt, wurde das Werk noch im selben Jahr von der katholischen Kirche wieder verboten und Galilei von der Inquisition 1633 zum Widerruf gezwungen. Doch war die Astronomie und die Erforschung des Sonnensystems nicht mehr aufzuhalten, obschon die katholische Kirche noch bis zum ersten Drittel des 18. Jahrhunderts brauchte, um die naturwissenschaftlichen Tatsachen zu akzeptieren.

Am Übergang zur Neuzeit dient die Sonne noch als Symbol des Absolutismus zur Rechtfertigung der Herrscher (Ludwig XIV als Sonnenkönig) und dieselbe Sonne wird von der Internationalen Arbeiterbewegung als Leitbild und Symbol bemüht, wenn gesungen wird: „Brüder, zur Sonne, zur Freiheit, Brüder, zum Lichte empor.“ Noch immer aber spendet die Sonne Licht und Wärme und ist die wesentliche Energiequelle für unsere Erde.

Die moderne Wissenschaft bringt es an den Tag. Das Universum entstand vor 15 Milliarden Jahren, das Sonnensystem vor 5 Milliarden und unsere Erde dann vor 4,5 Milliarden Jahren. Erste Lebensformen darauf entstanden vor 3,5 Milliarden Jahren und die Darwin'sche Evolution hatte diese ganze Zeitspanne zur Verfügung zur Artenentwicklung mit der enormen Vielfalt und hin bis zum Menschen, dem Homo sapiens sapiens, wie wir uns selbstbewusst nennen. Und nun geht es wieder los, in den USA, speziell in Pennsylvania, wo christliche Fundamentalisten gegen die Evolutionstheorie Sturm laufen und für eine wörtliche Auslegung der biblischen Schöpfungsgeschichte plädieren, die vor gerade mal 6000 Jahre ihren Anfang genommen habe. Der Versuch einer gerichtlichen Klärung erinnert in fataler Weise an die Inquisitionsbemü- 
hung zu Galilei’s Zeiten [3]. „Und sie bewegt sich doch!“, legen die Literaten ihm in den Mund, und sie strahlt schon 5 Milliarden Jahre, unsere Sonne, sei angefügt.

Eine neue Bedeutung erfährt die Sonne seit 150 Jahren. Befreit von religiösen Inhalten und ritueller Symbolik steht der Sonnengenuss für eine individuelle, selbst bestimmte Lebensgestaltung die zuweilen kultischen, ja revolutionären Charakter annimmt. Der Aufenthalt in frischer Luft und an der Sonne, Sonnenexposition und Sonnengenuss, werden Ausdruck von Selbstbestimmung, von Gesundheit und Schönheit, und stehen auch für persönliche Dynamik und Freizeit. Sonnenbaden und gebräunte Haut sind wieder in!

Sonnenfanatiker beschreiben besondere Formen von Sonnenkult. So Arnold Rikli (1823 - 1906), der im Jahre 1855 im damaligen Veldes im österreichischen Oberkrain (jetzt Bled in Slowenien) ein Sanatorium für kombinierte Sonnen- und Dampfbad-Therapie einrichtete. Biologen und Ärzte entdeckten die Effekte der Sonne zur Gesunderhaltung und zur Therapie von Hautkrankheiten, zur Prophylaxe der Rachitis sowie zur Unterstützung bei chronischen Infektionen. Die Heliotherapie wurde als Teil der Klimabehandlung im Hochgebirge und auf den küstennahen Inseln etabliert. 1903 erhielt der dänische Arzt Nils Riedberg Finsen (1860-1904) für die Lichtbehandlung der Hauttuberkulose (Lupus vulgaris) den Nobelpreis für Medizin. Die Photodermatologie entwickelt sich aus der Lichtbiologie heraus. Die natürliche Globalstrahlung und deren Imitation durch künstliche Lichtquellen sowie die Selektion besonders wirksamer Spektralbereiche und deren Kombination mit anderen Therapien und Verfahren führen zur dermatologischen Phototherapie und ihren gewaltigen Erfolgen [4].

Der unkontrollierte und unmäßige Sonnengenuss erfolgt seit der Mitte des 20. Jahrhunderts in der gewonnenen Freizeit und Freizügigkeit, mächtig gedrängt im Urlaub, und ergreift fast suchtartig große Teile der hellhäutigen Bevölkerung. Das „Alarmsignal“ des Sonnenbrandes wird erstaunlich gering eingeschätzt und nur ungenügend berücksichtigt (Abb. 2). Und mit Latenzzeiten von ein bis zwei Jahrzehnten haben wir die Bescherung: Der moderne fast fanatische Sonnenkult führt zur enormen Zunahme von lichtinduzierten Hautkarzinomen und zur vorzeitigen Hautalterung.

Die allermeisten Hautkarzinome erweisen sich als teilweise oder massiv Licht induziert und ihre Inzidenz hat sich in den vergangenen Jahrzehnten jeweils verdoppelt. Gegenwärtig werden jährlich an die 200 Patienten mit neu auftretenden Basaliomen und 30 mit neuen Spinaliomen pro 100000 Menschen gefunden und zudem jährlich 10-20 neue Melanome. Und ein Ende dieser Explosion ist noch keineswegs abzusehen. Therapie, Früherfassung und Prophylaxe sind vordringliche Programme, doch ist es sehr schwierig, den Sonnenhunger zu dämpfen und ein vernünftiges, also schadensarmes Sonnenverhalten der Bevölkerung zu erwirken.

Und die vorzeitige Hautalterung ist sichtbar und beeinträchtigt das Schönheitsbewusstsein massiv. Vergröberung der Hautstruktur, Fältchen und grobe Falten, plumpe Mimik sowie Verfärbungen und trockene Schuppung sind unverkennbar, auffällig und werden als hässlich, ja diskriminierend empfunden (Abb. 3). Dies schreckt erstaunlicherweise kaum vor Sonnengenuss $a b$, induziert aber eine Fülle von kosmetischen und invasiven Maßnahmen mit dem Ziel der Behandlung oder mindestens der Eindämmung der vorge-

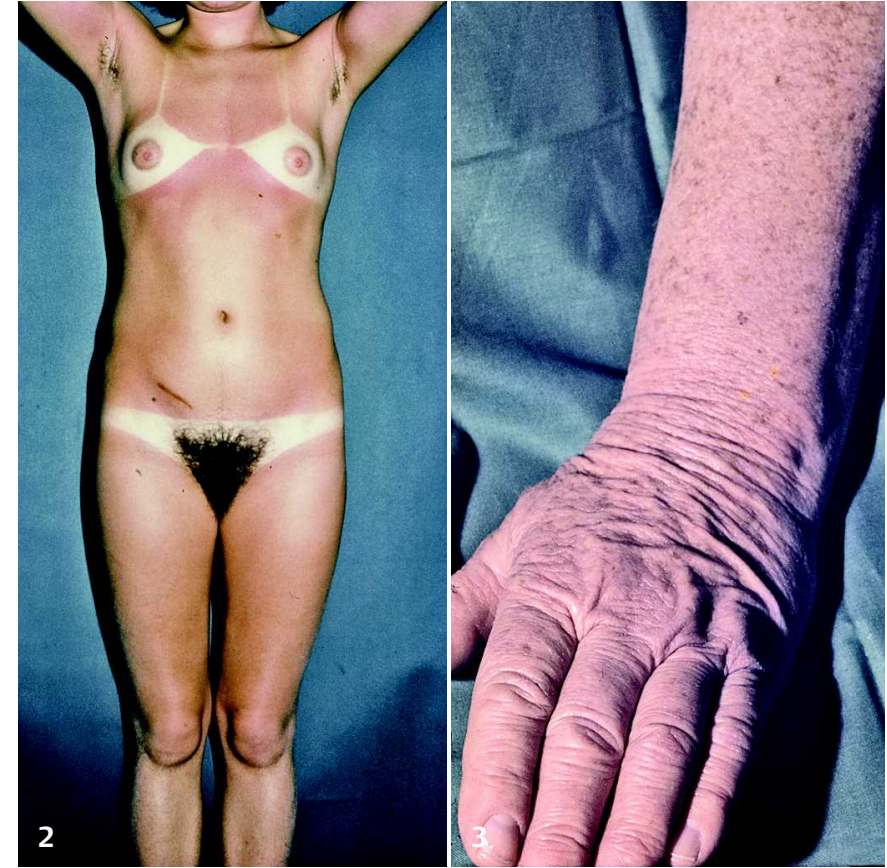

Abb. 2 Generalisierter Sonnenbrand (Lichtentzündung) $24 \mathrm{~h}$ nach übermäßiger Sonnenexposition. Ein oft nicht verstandenes Warnzeichen für die Überdosierung.

Abb. 3 Vorzeitige und lichtbedingte, bizarre Alterung der Haut: grobe Faltung am Handrücken und Fleckung des Unterarms.

zogenen und bizarr gestalteten Alterung der Haut. Anti-Aging ist das Schlagwort und betrifft auch alle anderen Organe des Körpers, auch das zentrale Nervensystem. Nun aber gehen die lichtbedingte Alterung der Haut, die schadensbedingte Abnützung der Organe und auch die neurodegenerativen Vorgänge alle mit entzündlichen Reaktionen einher und führen, teilweise wenigstens, zu vermehrt und vorzeitig auftretenden Tumoren. Es ist nicht unerwartet, dass moderne antientzündliche Wirkstoffe (Prostaglandin-Antagonisten vom Typ der magenfreundlichen COX-2-Hemmer) sog. Anti-aging-Effekte aufweisen oder wenigstens prophylaktische Wirkung zugesprochen bekommen. Allerdings ist der aufkommende Enthusiasmus, solche Stoffe bald als Medikamente einführen zu können, durch das gehäufte Auftreten von kardiovaskulären Zwischenfällen während der ausgedehnten klinischen Prüfungen kräftig gedämpft worden. Die Bäume wachsen nicht in den Himmel, oder wenigstens zur Zeit noch nicht. Nach wie vor gilt die Schadensmeidung, also kontrolliertes, gedrosseltes Sonnenverhalten, gesunde Kost, vernünftige Lebensführung als effektives und schadensfreies „Anti-Aging“. Und Gedächtnis-Training zur Alzheimer-Prophylaxe ist auch Anti-Aging.

\section{Literatur}

${ }^{1}$ Jung EG. Sonnenkultur und Sonnenkult. Ärztliche Kosmetologie 1989; 19: $114-116$

2 Bibel, AT: Psalm 19, 5-7

${ }^{3}$ Köhler A. Gott und Darwin vor Gericht. NZZ Nr. 271, 19./20. 11. 2005

${ }^{4}$ Jung EG. 75 Jahre Deutsche Gesellschaft für Lichtforschung. Akt Dermatol 2003; 29: $252-260$

Prof. Dr. E. G. Jung

Maulbeerweg 20

69120 Heidelberg 